\title{
Efeitos da facilitação neuromuscular proprioceptiva sobre variáveis cardiorrespiratórias: revisão narrativa da literatura
}

\author{
Effects of proprioceptive neuromuscular facilitation on cardiorespiratory
} variables: narrative literature review

Gustavo Silveira da Silva ${ }^{1}$ Natanyelle Steffen Freiberger ${ }^{\text {II }}$ Nathália Cristina Oliveira TEODORO

FERNANDA MACHADO ${ }^{\text {II }}$

KaROLINY DOS SANTOS ISOPPO 'Universidade de São PaUlo (USP), SÃo PAULO/SP - BRASIL "Universidade do Sul de SANTA Catarina (Unisul), Palhoça/ SC - BRASIL
Resumo A Facilitação Neuromuscular Proprioceptiva consiste em um conceito de tratamento com quatro mecanismos teóricos, referidos como inibição autogênica, inibição recíproca, relaxamento do estresse e a teoria do controle do portão, que aumentam a amplitude de movimento e a ativação muscular. Há muitos anos essa técnica é utilizada na fisioterapia, principalmente na área de reabilitação clínica. Contudo, não há na literatura uma revisão bibliográfica de sua aplicação na fisioterapia cardiorrespiratória. Este artigo de revisão da literatura tem por objetivo abordar os efeitos obtidos da utilização dessa técnica sobre as várias analisadas correspondentes aos sistemas cardiovascular e respiratório das amostras dos estudos. Tal revisão, por meio dos resultados obtidos, apoia o uso dessa técnica na prática clínica cardiorrespiratória por demonstrar efeitos positivos sobre os sintomas dos pacientes avaliados. Palavras-chave: Propriocepção; Modalidades de Fisioterapia; Reabilitação Cardíaca; Sistema Respiratório; Terapia POR EXERCícIO.

Abstract Proprioceptive Neuromuscular Facilitation consists of a treatment concept with four theoretical mechanisms, referred to as autogenic inhibition, reciprocal inhibition, stress relaxation, and the theory of gate control, which increase range of motion and muscle activation. This technique has been used in physiotherapy for many years, mainly in the area of cardiorespiratory physiotherapy. However, there is no literature review of its application in this area in the literature. This article of literature review aims to bring the effects obtained from the use of this technique on the several analyzed corresponding to the cardiovascular and respiratory systems of the study samples. This review, based on the results obtained, supports the use of this technique in clinical cardiorespiratory practice as it demonstrates positive effects on the symptoms of the patients evaluated.

Keywords: Proprioception, Physiotherapy Modalities, Cardiac Rehabilitation, Respiratory System, Exercise Therapy. 


\section{INTRODUÇÃO}

A Facilitação Neuromuscular Proprioceptiva (FNP) (Proprioceptive Neuromuscular Facilitation - PNF) consiste em um conceito de tratamento com quatro mecanismos teóricos, referidos como inibição autogênica, inibição recíproca, relaxamento do estresse e a teoria do controle do portão, que aumentam a amplitude de movimento e a ativação muscular. ${ }^{1}$ É baseado na filosofia de que todos os seres humanos têm o potencial subdesenvolvido que poderia ser usado de forma abrangente no nível físico e mental em pacientes com deficiências musculoesqueléticas. ${ }^{2}$ Ou seja, a filosofia de FNP é abordar não apenas as questões físicas dos pacientes com deficiências nas estruturas do corpo e funções do corpo que causam dor ou limitação de atividades, mas também na abordagem de corpo total e na abordagem positiva para se concentrar em melhorar o nível de atividade e as estruturas e funções do corpo. ${ }^{1,2}$

Esse método utiliza movimentos em diagonais e espirais, tendo como objetivo a facilitação, o fortalecimento, o ganho de controle e de coordenação dos movimentos. Ativa ainda proprioceptores que se localizam nas articulações, tendões e músculos que, consequentemente, melhoram a função motora. ${ }^{3 "}$ $\mathrm{O}$ alongamento por FNP caracteriza-se pelo uso de contração muscular ativa com o objetivo de ocasionar inibição autogênica do músculo alongado. Quando aplicada, ocorre relaxamento muscular reflexo que, associada com alongamento passivo, promove aumento no ganho da amplitude de movimento. ${ }^{4}$

Muitos estudos demonstram os efeitos benéficos da utilização de FNP nos atendi- mentos fisioterapêuticos como na mobilidade torácica, ${ }^{5}$ saturação de oxigênio, ${ }^{6}$ pressão arterial, ${ }^{7}$ percepção de dispneia, ${ }^{8}$ capacidade vital, ${ }^{9}$ fadiga muscular, ${ }^{10}$ função pulmonar, ${ }^{11}$ e redução no tempo de intubação e permanência na UTI. ${ }^{12}$ Além disso, por ser uma técnica de fácil aplicação e de baixo custo seu incremento nos tratamentos fisioterapêuticos torna-se viável e interessante. Contudo, apesar de nas duas últimas décadas a utilização de FNP no campo da fisioterapia cardiorrespiratória ter aumentado ${ }^{5,7,13}$ nenhuma revisão de literatura sobre o tema foi realizada até o momento.

Revisar as publicações sobre o tema e trazer informações sobre a aplicabilidade e resultados obtidos pelo FNP nessa área auxilia os profissionais da saúde a conhecer melhor a técnica e integrá-la ainda mais em suas intervenções. Sendo assim, o objetivo deste estudo foi apresentar uma revisão de literatura sobre os efeitos de FNP em variáveis do sistema cardiovascular e respiratório e sua possível indicação nessa área clínica por meio da fisioterapia.

\section{Metodologia}

Este artigo é uma revisão narrativa da literatura e teve como banco de dados o PubMed, SciELO, Google Acadêmico, Scopus e MedLine para a busca de artigos publicados entre os meses de janeiro de 1999 e abril de 2021. Os conceitos base da área de facilitação neuromuscular proprioceptiva referidos em estudos sobre o sistema cardiorrespiratório foram aplicados para a busca dos artigos. Dessa forma, as palavras-chave utilizadas foram: PNF and "respiratory system", $P N F$ and "cardiac rehabilitation", PNF and 
"cardiovascular system", PNF and "respiratory muscles", PNF and "respiration", PNF and "thoracic mobility", PNF and "diaphragmatic liberation", PNF and "pulmonary function", $P N F$ and "breathing exercises", PNF and "respiration excercise" e PNF and "lung function". Assim como seus correspondentes nas línguas espanhola e portuguesa.

A pesquisa foi limitada às línguas inglesa, espanhola e portuguesa. Como critérios de inclusão para esta revisão os estudos deveriam ser realizados em humanos com idade $\geq 18$ anos e que tinham sido publicados entre os anos de 1999 e 2021. Os critérios de exclusão adotados foram: estudos pré-clínicos, dissertações ou teses acadêmicas, cartas editoriais ou resumos publicados em congressos. Ao final da coleta de dados foi realizada uma análise de títulos e resumos para obtenção de artigos potencialmente relevantes para a revisão.

A qualidade dos estudos foi avaliada pela escala de qualidade de PEDro. O objetivo dessa escala é auxiliar os leitores na interpretação quanto a qualidade metodológica dos estudos controlados e aleatorizados (ECAs) (validade interna, critérios 2 a 9 da escala), bem como avaliar a descrição estatística, isto é, se o estudo contém informações estatísticas mínimas para que os resultados possam ser interpretáveis (critérios 10 e 11 da escala). Não são avaliadas a validade externa do estudo, generalização dos resultados, nem a magnitude do efeito de tratamento (isto é, se os resultados são clinicamente relevantes ou não). Os avaliadores da PEDro analisam somente o que está reportado no manuscrito, quando há dúvida por parte do avaliador na hora de pontuar o critério, ele o classifica como "não". A pon- tuação final da escala de qualidade PEDro é dada por meio da soma do número de critérios que foram classificados como satisfatórios entre os critérios 2 e 11 . O critério 1 não é considerado para a pontuação final por tratar-se de um item que avalia a validade externa do estudo. ${ }^{14}$ Cada ECA foi avaliado por quatro avaliadores independentes e, no caso de discordância de avaliação de qualquer item em questão, um quinto avaliador fez uma arbitragem final. A pontuação final pode variar entre 0 e 10 pontos. Sendo a nota 0 uma péssima pontuação e 10 uma excelente pontuação. ${ }^{14}$

\section{RESUltados E discussão}

Foram identificados 2.793 artigos, sendo que 1.645 foram selecionados para triagem após retirada dos títulos repetidos. Após leitura dos títulos e resumos, 19 artigos foram lidos na íntegra e quatro deles foram retirados por estarem nos critérios de exclusão. Ao final, 15 estudos foram selecionados por enquadrarem-se nos critérios preestabelecidos (Figura 1).

Sobre as bases de dados incluídas na pesquisa, 10 artigos foram localizados nas bases de dados indexadas no Portal de Periódicos da $\mathrm{CAPES}^{5-13,15}$, quatro em literatura cinzenta (Google Acadêmico) ${ }^{16-19} \mathrm{e}$ um na base ScieLo ${ }^{20}$. Sete dos estudos incluídos são originários do Brasil $(46,6 \%)$ e oito estudos são internacionais $(53,4 \%)$, entretanto o país com maior número de estudos nessa área foi o Brasil. Quanto a qualidade metodológica dos estudos obtida pela escala PEDro, foi demonstrado que nove dos 15 estudos alcançaram uma pontuação $\geq 6$ pontos (Tabela 1). 


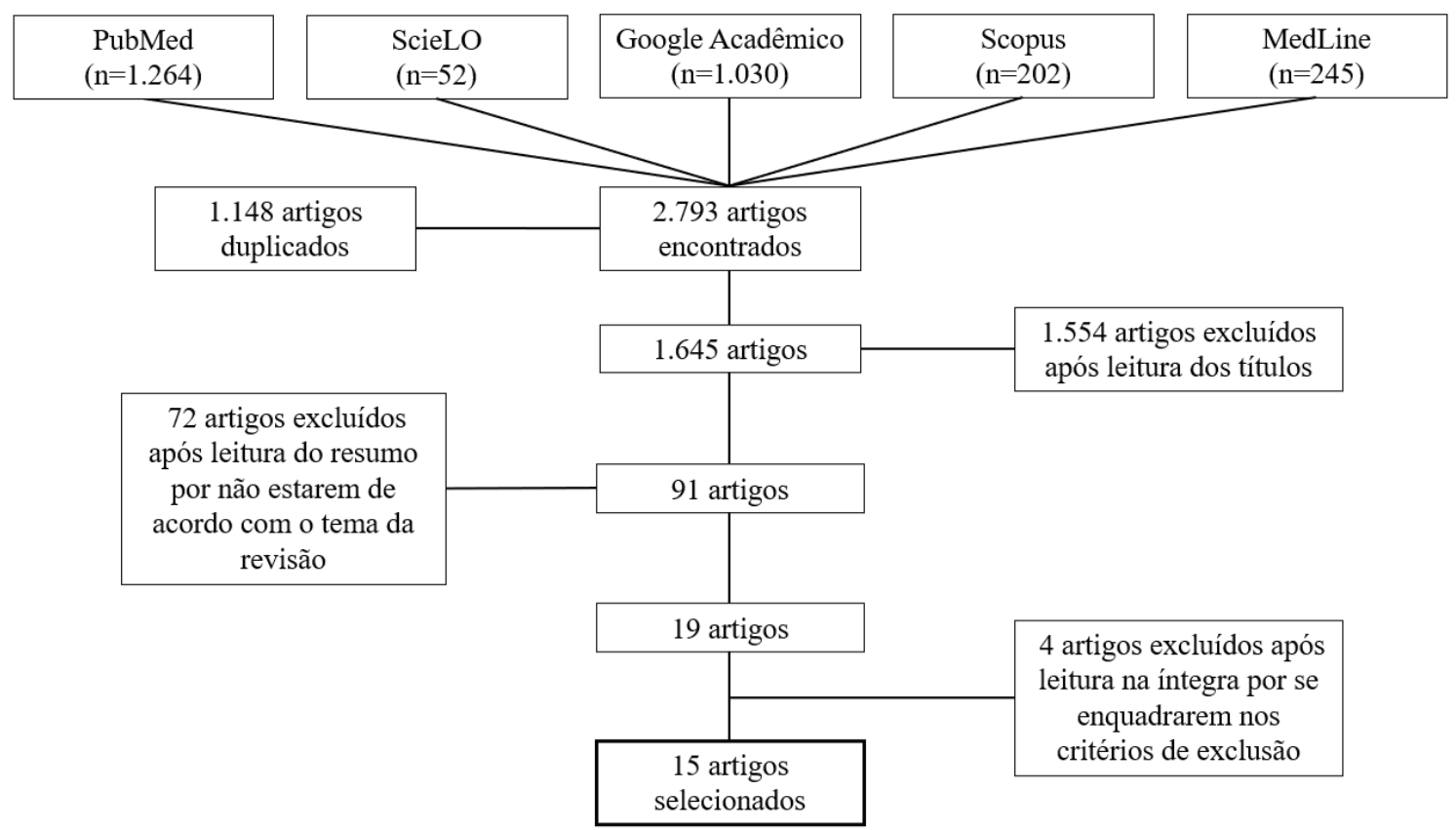

Figura 1. Fluxograma da seleção dos artigos.

Tabela 1. Países de origem dos artigos e suas pontuações pela escala PEDro.

\begin{tabular}{c|c|c}
\hline Autor (ano) & País de origem & $\begin{array}{c}\text { Pontuação escala PEDro (0 } \\
-10)\end{array}$ \\
\hline Nitz e Burke $(2002)^{5}$ & Austrália & $3 / 10$ \\
\hline Moreno et. al. $(2009)^{20}$ & Brasil & $9 / 10$ \\
\hline Cortopassi et. al. $(2009)^{6}$ & Brasil & $4 / 10$ \\
\hline Souza et. al. $(2010)^{7}$ & Brasil & $4 / 10$ \\
\hline Velloso et. al. $(2013)^{8}$ & Brasil & $4 / 10$ \\
\hline Morano et. al. $(2013)^{12}$ & Brasil & $9 / 10$ \\
\hline Silva et. al. $(2013)^{17}$ & Brasil & $6 / 10$ \\
\hline Seo e Cho $(2014)^{9}$ & Coreia do Sul & $6 / 10$ \\
\hline Reddy et. al. $(2014)^{18}$ & Índia & $4 / 10$ \\
\hline Silva et. al. $(2014)^{16}$ & Brasil & $6 / 10$ \\
\hline Br e Goud $(2017)^{19}$ & Índia & $6 / 10$ \\
\hline Pyszora et. al. $(2017)^{10}$ & Polônia & $5 / 10$ \\
\hline Kim e Lee $(2017)^{11}$ & Coreia do Sul & $6 / 10$ \\
\hline Bujar-Misztal e Chciałowski $(2018)^{15}$ & Polônia & $6 / 10$ \\
\hline Slupska et. al. $(2019)^{13}$ & Polônia & $6 / 10$ \\
\hline
\end{tabular}


A Tabela 2, para melhor visibilidade dos dados obtidos, apresenta em ordem cronológica os estudos tratados nesta revisão e suas características, como o tipo de estudo e intervenção, principais variáveis e resultados obtidos.

A maioria dos estudos foi composta por randomização dos participantes entre grupos de intervenção e controle e apenas cinco foram quase experimentais. Nos estudos selecionados, as intervenções de FNP foram aplicadas na distrofia miotônica, ${ }^{5}$ doença pulmonar obstrutiva crônica (DPOC), ${ }^{6-8}$ pré e pós-operatório de ressecção de câncer de mama, ${ }^{12}$ envenenamento por organofosforados, ${ }^{18}$ câncer em estado avançado, ${ }^{10}$ dor lombar crônica, ${ }^{11}$ pós-cirurgia de ponte de safena, ${ }^{15}$ pós-AVC isquêmico, ${ }^{13}$ atletas de natação ${ }^{17}$ e em indivíduos jovens e idosos saudáveis. ${ }^{9,12,16}$ and pulmonary complications assessed after lung cancer resection (phase 2

Em quase metade dos estudos o FNP foi utilizado como tratamento único no grupo experimental. Nos demais, foi associado com outras intervenções, dentre elas: expansão pulmonar ensinada ${ }^{5}$, caminhada em esteira ${ }^{6}$, alongamentos de musculatura respiratória ${ }^{6}$, fisioterapia respiratória convencional ${ }^{18}$, liberação miofascial ${ }^{10}$ ou eletroterapia ${ }^{11}$.

Os resultados obtidos em um estudo com pacientes com distrofia muscular permitem observar que o FNP aumentou a saturação de oxigênio sanguíneo arterial $\left(\mathrm{SO}_{2}\right)$ e da mobilidade torácica e diminuição de $20 \%$ da frequência respiratória (FR). ${ }^{5}$

As distrofias musculares são doenças primárias, hereditárias, progressivas e degenerativas do músculo esquelético. Elas causam fraqueza progressiva que geralmente começa com uma distribuição característica (por exemplo, cintura-membro, faciosca- pulohumeral, oculofaríngea). As alterações patológicas no músculo esquelético são degenerativas, muitas vezes com aspecto distrófico característico (fibras degenerativas e regenerativas, variação no diâmetro das fibras, substituição por gordura e tecido conjuntivo). ${ }^{21}$

Talvez a principal explicação para os resultados obtidos neste estudo podem ser pelo protocolo implementado pelos pesquisadores, no qual a alternância de posições dos pacientes poderia redirecionar o ar para os campos pulmonares basais e as técnicas de FNP aumentarem o recrutamento da musculatura inspiratória. ${ }^{2,5}$

$\mathrm{A} \mathrm{SO}_{2}$ e FR também foram melhoradas em pacientes com DPOC, assim como os níveis de gás carbônico sanguíneo $\left(\mathrm{CO}_{2}\right)$, melhora da ventilação pulmonar, frequência cardíaca (FC), pressão arterial (PA) e níveis de lactato. ${ }^{7}$

É bem conhecido que pacientes com DPOC apresentam alterações no tipo de fibra muscular, redução da capilaridade, diminuição da enzima oxidativa, depleção nutricional e uso de esteroides. Juntos, eles podem explicar potencialmente a redução da resistência muscular. ${ }^{22,23}$ A perda de peso e o desgaste muscular há muito são considerados eventos que destacam a progressão da DPOC. ${ }^{6} \mathrm{O}$ exercício pode ser a primeira opção em pacientes estáveis para aumentar a massa muscular e melhorar a resistência ao exercício. ${ }^{8}$ Os movimentos de FNP são baseados em princípios fisiológicos e seguem a anatomia das fibras musculares, além de recrutarem um grande número muscular, principalmente os da cintura escapular. ${ }^{6,8} \mathrm{O}$ treinamento com movimentos diagonais é eficaz para aumentar a força dos membros superiores e 
fortalecer a cintura escapular de pacientes com DPOC. ${ }^{6}$

Quanto aos valores de lactato no pico do exercício de braço dominante foram menores nos pacientes que receberam FNP do que os do grupo de exercícios convencionais. ${ }^{7}$ Talvez isso se deva pelo fato de os testes terem sido realizados exclusivamente com o membro superior dominante dos participantes ao contrário de outro estudo que submeteu seus pacientes a um teste incremental em ambos os braços. ${ }^{24}$ Provavelmente isso reflete aos achados de uma menor elevação de variáveis como produção de lactato muscular devido à menor massa muscular envolvida no exercício. ${ }^{7}$

Por outro lado, técnicas de FNP em pacientes envenenados por organofosfo- rados, não obtiveram alteração dos níveis de $\mathrm{PA}, \mathrm{FC}, \mathrm{FR}$ e $\mathrm{SO}_{2}$, após intervenção. ${ }^{18}$ Provavelmente, os resultados não foram favoráveis, pois esses compostos tóxicos são inibidores da enzima acetilcolinesterase que bloqueia a transmissão neuromuscular. ${ }^{25}$ Organofosforados causam toxicidade aguda ao sistema nervoso central (SNC), que resulta em insuficiência respiratória. ${ }^{25,26} \mathrm{~A}$ área intermediária da superfície ventral da medula oblonga parece ser o local mais sensível para a depressão do SNC. ${ }^{25}$ O FNP atua nas vias neuromusculares periféricas intactas, sendo assim as técnicas não contribuíram com a melhora do quadro dos pacientes devido ao comprometimento das vias neuromusculares periféricas e centrais. ${ }^{18}$ 
Tabela 2. Características dos artigos selecionados.

\begin{tabular}{|c|c|c|c|c|}
\hline $\begin{array}{l}\text { Autor } \\
\text { (ano) }\end{array}$ & $\begin{array}{l}\text { Tipo de estudo e } \\
\text { amostra }\end{array}$ & Tipo de intervenção & $\begin{array}{l}\text { Principais } \\
\text { variáveis } \\
\text { analisadas }\end{array}$ & $\begin{array}{l}\text { Resultados } \\
\text { obtidos }\end{array}$ \\
\hline Nitz e Burke $(2002)^{5}$ & $\begin{array}{l}\text { Randomizado duplo } \\
\text { cego } \\
\text { Distrofia miotônica } \\
(\mathrm{n}=7)\end{array}$ & $\begin{array}{l}\text { Descansando com } \\
\text { apoio elevado } \\
\text { sentado; descansando } \\
\text { deitado no lado } \\
\text { esquerdo; FNP de } \\
\text { respiração profunda } \\
\text { com apoio elevado; } \\
\text { FNP de respiração } \\
\text { profunda deitado } \\
\text { no lado esquerdo; } \\
\text { expansão pulmonar } \\
\text { ensinada (SBE) em } \\
\text { posição de apoio } \\
\text { elevado e SBE em } \\
\text { posição lateral } \\
\text { esquerda. }\end{array}$ & $\begin{array}{l}\text { Saturação } \\
\text { de oxigênio } \\
\text { arterial }\left(\mathrm{SO}_{2}\right) \text {, } \\
\text { frequência } \\
\text { cardíaca }(\mathrm{FC}) \text {, } \\
\text { movimento } \\
\text { tóraco- } \\
\text { abdominal } \\
\text { e frequência } \\
\text { respiratória } \\
\text { (FR) }\end{array}$ & $\begin{array}{l}\text { A intervenção com } \\
\text { FNP aumentou } \\
\text { a saturação de } \\
\text { oxigênio periférica } \\
\text { e mobilidade } \\
\text { tóraco-abdominal } \\
\text { e diminuiu } \\
\text { a frequência } \\
\text { respiratória em } \\
\text { torno de } 20 \% .\end{array}$ \\
\hline $\begin{array}{l}\text { Moreno et. al. } \\
(2009)^{20}\end{array}$ & $\begin{array}{l}\text { Randomizado e } \\
\text { controlado } \\
\text { Adultos saudáveis } \\
\text { Grupo FNP }(\mathrm{n}=12) \\
\text { e Grupo controle } \\
(\mathrm{n}=12)\end{array}$ & $\begin{array}{l}\text { Grupo FNP = } \\
\text { exercícios de FNP. } \\
\text { Grupo controle } \\
=\text { exercícios de } \\
\text { membros superiores } \\
\text { direito } \\
\text { e esquerdo nas } \\
\text { diagonais do método } \\
\text { Kabat }\end{array}$ & $\begin{array}{l}\text { Cirtometria } \\
\text { axilar e } \\
\text { xifoideana }\end{array}$ & $\begin{array}{l}\text { FNP aumentou } \\
\text { a cirtometria } \\
\text { axilar e xifoideana } \\
\text { comparado ao } \\
\text { grupo controle } \\
\text { que não possuiu } \\
\text { melhoras } \\
\text { significativas. }\end{array}$ \\
\hline $\begin{array}{l}\text { Cortopassi et. al. } \\
(2009)^{6}\end{array}$ & $\begin{array}{l}\text { Quase experimental } \\
\text { DPOC }(\mathrm{n}=71)\end{array}$ & $\begin{array}{l}\text { Esteira + FNP + } \\
\text { alongamentos ou } \\
\text { respiração }\end{array}$ & $\begin{array}{l}\text { PImáx, PEmáx, } \\
\text { dispneia }\end{array}$ & $\begin{array}{l}\text { Melhora de PImáx, } \\
\text { PEmáx e percepção } \\
\text { de dispneia. }\end{array}$ \\
\hline Souza et. al. $(2010)^{7}$ & $\begin{array}{l}\text { Quase experimental } \\
\text { DPOC }(\mathrm{n}=16)\end{array}$ & $\begin{array}{l}\text { Exercícios com os } \\
\text { membros superiores } \\
\text { sem suporte (FNP) }\end{array}$ & $\begin{array}{l}\text { Consumo } \\
\text { de oxigênio, } \\
\text { produção de } \\
\text { monóxido de } \\
\text { carbono }\left(\mathrm{CO}_{2}\right) \text {, } \\
\text { FR, ventilação } \\
\text { pulmonar, FC } \\
\text { pressão arterial } \\
\text { (PA) e níveis } \\
\text { de lactato } \\
\text { sanguíneos }\end{array}$ & $\begin{array}{l}\text { Houve melhora } \\
\text { significativa de } \\
\text { todas as variáveis } \\
\text { analisadas após } \\
\text { treinamento } \\
\text { com membros } \\
\text { superiores sem } \\
\text { suporte associado } \\
\text { com a FNP }\end{array}$ \\
\hline Velloso et. al. $(2013)^{8}$ & $\begin{array}{l}\text { Quase experimental } \\
\text { DPOC }(\mathrm{n}=35)\end{array}$ & $\begin{array}{l}\text { FNP em membros } \\
\text { superiores }\end{array}$ & FR e dispneia & $\begin{array}{l}\text { Houve diminuição } \\
\text { da frequência } \\
\text { respiratória e } \\
\text { dispneia após } \\
\text { intervenção. }\end{array}$ \\
\hline
\end{tabular}




\begin{tabular}{|c|c|c|c|c|}
\hline $\begin{array}{l}\text { Autor } \\
\text { (ano) }\end{array}$ & $\begin{array}{l}\text { Tipo de estudo e } \\
\text { amostra }\end{array}$ & Tipo de intervenção & $\begin{array}{l}\text { Principais } \\
\text { variáveis } \\
\text { analisadas }\end{array}$ & $\begin{array}{l}\text { Resultados } \\
\text { obtidos }\end{array}$ \\
\hline $\begin{array}{l}\text { Morano et. al. } \\
(2013)^{12}\end{array}$ & $\begin{array}{l}\text { Randomizado } \\
\text { simples cego } \\
\text { Pré e pós-operatório } \\
\text { de ressecção de } \\
\text { câncer de pulmão } \\
\text { RP }(n=12) \text { e } \\
\text { Fisioterapia } \\
\text { respiratória }(n=12)\end{array}$ & $\begin{array}{l}\mathrm{RP}=\text { treinamento } \\
\text { de força e resistência } \\
\text { de membros } \\
\text { superiores por meio } \\
\text { de FNP; Fisioterapia } \\
\text { respiratória } \\
=\text { exercícios } \\
\text { respiratórios para } \\
\text { expansão pulmonar }\end{array}$ & $\begin{array}{l}\text { PImáx, PEmáx, } \\
\text { capacidade vital } \\
\text { forçada, teste } \\
\text { de caminhada } \\
\text { de } 6 \text { minutos, } \\
\text { morbidade } \\
\text { respiratória, } \\
\text { tempo de } \\
\text { permanência } \\
\text { e tempo de } \\
\text { intubação }\end{array}$ & $\begin{array}{l}\text { RP no pré- } \\
\text { operatório: } \\
\text { melhorou de forma } \\
\text { significativa as } \\
\text { variáveis analisadas; } \\
\text { Pós-operatório: } \\
\text { diminuição } \\
\text { do tempo de } \\
\text { intubação, } \\
\text { morbidade } \\
\text { pulmonar e } \\
\text { permanência no } \\
\text { leito. }\end{array}$ \\
\hline $\begin{array}{l}\text { Costa e Silva et. al. } \\
(2013)^{17}\end{array}$ & $\begin{array}{l}\text { Randomizado e } \\
\text { controlado } \\
\text { Atletas femininas } \\
\text { Grupo SS }(\mathrm{n}=4) \\
\text { Grupo FNP }(\mathrm{n}=4) \\
\text { Grupo controle } \\
(\mathrm{n}=4)\end{array}$ & $\begin{array}{l}\text { SS: } 2 \text { séries de } \\
30 \text { segundos de } \\
\text { alongamento dos } \\
\text { músculos peitorais } \\
\text { e bíceps ( } 15 \text { seg. } \\
\text { de descanso). } \\
\text { FNP: } 2 \text { séries } \\
\text { de alongamento } \\
\text { músculos peitorais } \\
\text { e bíceps, com } \\
6 \text { segundos } \\
\text { de contração } \\
\text { isométrica seguidos } \\
\text { de } 24 \text { segundos } \\
\text { de alongamento } \\
\text { sustentado (15 seg. } \\
\text { de descanso). } \\
\text { Grupo controle não } \\
\text { houve intervenção. }\end{array}$ & $\mathrm{PA}, \mathrm{FC} \mathrm{e} \mathrm{SO}{ }_{2}$ & $\begin{array}{l}\text { Não houve } \\
\text { alteração nas } \\
\text { pressões sistólica e } \\
\text { diastólica e FC. } \\
\text { Houve redução } \\
\text { significativa de } \mathrm{SO}_{2} \\
\text { nos grupos SS e } \\
\text { FNP comparado ao } \\
\text { grupo controle. }\end{array}$ \\
\hline Seo e Cho $(2014)^{9}$ & $\begin{array}{l}\text { Randomizado e } \\
\text { controlado } \\
\text { Adultos saudáveis } \\
\text { Grupo experimental } \\
(\mathrm{n}=14) \text { Grupo } \\
\text { controle }(\mathrm{n}=14)\end{array}$ & $\begin{array}{l}\text { Grupo experimental } \\
=\text { exercícios } \\
\text { respiratórios } \\
\text { associados com } \\
\text { FNP. Grupo } \\
\text { controle = exercícios } \\
\text { de respiração } \\
\text { diafragmática }\end{array}$ & $\begin{array}{l}\text { Volume } \\
\text { de reserva } \\
\text { expiratório e } \\
\text { capacidade vital }\end{array}$ & $\begin{array}{l}\text { O grupo } \\
\text { experimental } \\
\text { apresentou } \\
\text { melhora na } \\
\text { função pulmonar } \\
\text { comparado ao } \\
\text { grupo controle. }\end{array}$ \\
\hline Reddy et. al. $(2014)^{18}$ & $\begin{array}{l}\text { Quase experimental } \\
\text { Envenenamento por } \\
\text { organofosforado } \\
\text { Fisioterapia } \\
\text { respiratória e após } \\
3 \text { minutos FNP } \\
(n=30)\end{array}$ & $\begin{array}{l}\text { Fisioterapia } \\
\text { respiratória } \\
\text { convencional } \\
\text { = Vibrações, } \\
\text { percussões, } \\
\text { drenagem postural e } \\
\text { aspiração se indicado } \\
\text { + FNP de MMSS e } \\
\text { tórax }\end{array}$ & $\begin{array}{l}\text { Ventilação por } \\
\text { minuto, } \mathrm{SO}_{2}, \mathrm{FC} \\
\text { e PA }\end{array}$ & $\begin{array}{l}\text { FNP não aumentou } \\
\text { significativamente } \\
\text { os valores dos } \\
\text { parâmetros } \\
\text { pulmonares e } \\
\text { hemodinâmicos. }\end{array}$ \\
\hline Silva et. al. $(2014)^{16}$ & $\begin{array}{l}\text { Quase experimental } \\
\text { Adultos saudáveis } \\
(\mathrm{n}=10)\end{array}$ & $\begin{array}{l}\text { Teste de três séries } \\
\text { de uma repetição } \\
\text { máxima de exercícios } \\
\text { de membro superior } \\
\text { com a técnica de FNP } \\
\text { associado a polia }\end{array}$ & PA e FC & $\begin{array}{l}\text { Não houve } \\
\text { aumento de PA } \\
\text { e FC ao final da } \\
\text { intervenção }\end{array}$ \\
\hline
\end{tabular}




\begin{tabular}{|c|c|c|c|c|}
\hline $\begin{array}{l}\text { Autor } \\
\text { (ano) }\end{array}$ & $\begin{array}{l}\text { Tipo de estudo e } \\
\text { amostra }\end{array}$ & Tipo de intervenção & $\begin{array}{l}\text { Principais } \\
\text { variáveis } \\
\text { analisadas }\end{array}$ & $\begin{array}{l}\text { Resultados } \\
\text { obtidos }\end{array}$ \\
\hline Br e Goud (2017) $)^{19}$ & $\begin{array}{l}\text { Randomizado e } \\
\text { controlado } \\
\text { Idosos saudáveis } \\
\text { Grupo FNP }(n=20) \\
\text { Grupo alongamento } \\
\text { muscular }(n=20)\end{array}$ & $\begin{array}{l}\text { Grupo FNP }= \\
\text { técnica aplicada } \\
\text { para alongamento } \\
\text { da musculatura } \\
\text { respiratória. Grupo } \\
\text { alongamento } \\
\text { muscular = } \\
\text { alongamentos } \\
\text { convencionais } \\
\text { das musculaturas } \\
\text { respiratórias. }\end{array}$ & VEF1 e CVF & $\begin{array}{l}\text { Ambas as técnicas } \\
\text { melhoram } \\
\text { significativamente } \\
\text { a expansão } \\
\text { torácica e a função } \\
\text { pulmonar. }\end{array}$ \\
\hline $\begin{array}{l}\text { Pyszora et. al. } \\
(2017)^{10}\end{array}$ & $\begin{array}{l}\text { Randomizado e } \\
\text { controlado } \\
\text { Câncer em estado } \\
\text { avançado. } \\
\text { Grupo tratado } \\
(\mathrm{n}=30) \text { e Grupo } \\
\text { controle }(\mathrm{n}=30)\end{array}$ & $\begin{array}{l}\text { Grupo tratado }= \\
\text { exercícios ativos }+ \\
\text { liberação miofascial } \\
\text { + FNP; Grupo } \\
\text { controle não se } \\
\text { exercitou }\end{array}$ & $\begin{array}{l}\text { Escores de } \\
\text { fadiga e } \\
\text { percepção de } \\
\text { bem-estar geral }\end{array}$ & $\begin{array}{l}\text { O grupo tratado } \\
\text { apresentou } \\
\text { melhoras } \\
\text { significativas nos } \\
\text { escores de fadiga } \\
\text { e bem-estar geral } \\
\text { comparado ao } \\
\text { grupo controle. }\end{array}$ \\
\hline Kim e Lee $(2017)^{11}$ & $\begin{array}{l}\text { Randomizado e } \\
\text { controlado } \\
\text { Dor lombar crônica } \\
\text { Grupo fisioterapia } \\
\text { convencional } \\
(\mathrm{n}=15) \text { FNP + } \\
\text { AMST }(n=15)\end{array}$ & $\begin{array}{l}\text { Fisioterapia } \\
\text { convencional = bolsa } \\
\text { de água quente + } \\
\text { ultrassonografia } \\
\text { + corrente } \\
\text { interferencial. } \\
\text { FNP + AMST = } \\
\text { alongamentos } \\
\text { da musculatura } \\
\text { abdominal + técnicas } \\
\text { de FNP }\end{array}$ & $\begin{array}{l}\text { Função } \\
\text { pulmonar, dor } \\
\text { e índice de } \\
\text { incapacidade } \\
\text { funcional }\end{array}$ & $\begin{array}{l}\text { FNP + AMST } \\
\text { aumentou a } \\
\text { função pulmonar, } \\
\text { diminuiu a dor } \\
\text { e o índice de } \\
\text { incapacidade } \\
\text { funcional } \\
\text { comparado ao } \\
\text { grupo fisioterapia } \\
\text { convencional. }\end{array}$ \\
\hline $\begin{array}{l}\text { Bujar-Misztal e } \\
\text { Chciałowski }(2018)^{15}\end{array}$ & $\begin{array}{l}\text { Randomizado e } \\
\text { controlado } \\
\text { Pós cirurgia ponte } \\
\text { de safena } \\
\text { Grupo FNP }(n=22) \\
\text { Grupo controle } \\
(n=20)\end{array}$ & $\begin{array}{l}\text { Grupo FNP } \\
=\text { exercícios } \\
\text { respiratórios e } \\
\text { proprioceptivos da } \\
\text { respiração Grupo } \\
\text { controle = treino } \\
\text { dos músculos } \\
\text { respiratórios }\end{array}$ & $\begin{array}{l}\text { Relação VEF1/ } \\
\text { CVF e a } \\
\text { proporção da } \\
\text { capacidade } \\
\text { residual para } \\
\text { a capacidade } \\
\text { pulmonar total } \\
\text { (\% CPT de VR) }\end{array}$ & $\begin{array}{l}\text { Ambas as técnicas } \\
\text { obtiveram, na } \\
\text { mesma proporção, } \\
\text { melhoras } \\
\text { significativas } \\
\text { nas variáveis } \\
\text { analisadas. }\end{array}$ \\
\hline $\begin{array}{l}\text { Slupska et. al. } \\
(2019)^{13}\end{array}$ & $\begin{array}{l}\text { Randomizado e } \\
\text { controlado } \\
\text { Pós AVC isquêmico } \\
\text { Grupo FNP }(n=30) \\
\text { Grupo controle } \\
(n=30)\end{array}$ & $\begin{array}{l}\text { Grupo FNP = } \\
\text { estimulação } \\
\text { diafragmática e } \\
\text { da musculatura } \\
\text { intercostal. Grupo } \\
\text { controle = mudança } \\
\text { de posturas }\end{array}$ & $\begin{array}{l}\text { Eletromiografia } \\
\text { da musculatura } \\
\text { respiratória } \\
\text { acessória }\end{array}$ & $\begin{array}{l}\text { A FNP diminuiu } \\
\text { a atividade da } \\
\text { musculatura } \\
\text { acessória } \\
\text { comparado ao } \\
\text { grupo controle. }\end{array}$ \\
\hline
\end{tabular}

Legenda: FNP: Facilitação neuromuscular proprioceptiva; FC: frequência cardíaca; FR: frequência respiratória; PA: pressão arterial; $\mathrm{SO}_{2}$ : saturação de oxigênio arterial; DPOC: Doença pulmonar obstrutiva crônica; RP: Reabilitação pulmonar; SS: alongamento estático; PImáx: Pressão inspiratória máxima; PEmáx: Pressão expiratória máxima; VEF1: Volume expiratório forçado no primeiro segundo; CVF: Capacidade vital forçada; AMST: Alongamento muscular abdominal; CPT de VR: Proporção da capacidade residual para a capacidade pulmonar total; AVC: Acidente vascular cerebral. 
Outros estudos mostraram que a intervenção associada de FNP em pacientes com DPOC melhorou a percepção de dispneia e as pressões inspiratória máxima (PImáx) e expiratória máxima (PEmáx). ${ }^{6,8}$ Essas últimas variáveis de pressão também foram melhoradas no pré-operatório de ressecção de câncer de pulmão, assim como a capacidade vital forçada, e no teste de caminhada de 6 minutos. ${ }^{12}$ Já no pós-operatório houve redução da morbidade respiratória, do tempo de intubação e permanência na UTI, comparado ao grupo que recebeu apenas fisioterapia respiratória convencional. ${ }^{12}$ Os mecanismos de ação de FNP para esses resultados ainda não estão claros, mas se poderia atribuir esses benefícios à reabilitação com o uso rotineiro do treinamento muscular, conforme os resultados do estudo de Hulzebos et. al. $^{27}$ que mostraram que o treinamento da musculatura inspiratória antes da cirurgia torácica pode melhorar a PImáx pré-operatória em pacientes submetidos à cirurgia de revascularização do miocárdio.

As técnicas de FNP associadas com outras intervenções reduziu a fadiga em pacientes com câncer avançado e dor lombar crônica, além disso, houve melhora do bem-estar geral e aumento da capacidade e função pulmonar nesses grupos, respectivamente. ${ }^{10,11}$ A capacidade vital pulmonar e o volume de reserva expiratório também foram aumentados em adultos saudáveis. ${ }^{9}$

Uma população de pacientes com diagnóstico de câncer avançado que recebem cuidados paliativos necessitam de uma seleção de terapias específicas que deve ser baseada em uma análise individual cuidadosa do potencial e das limitações de determinados pacientes. Eles tendem a ser relutantes em realizar atividades físicas, dando razões como condicionamento físico e capacidade funcional limitada. ${ }^{28}$ Portanto, o programa de fisioterapia deve incluir tanto os elementos do tratamento que não requerem grandes gastos de energia por parte dos pacientes quanto os métodos que os envolvem ativamente. Sendo assim, programas com técnicas de relaxamento (relaxamento miofascial, estimulação respiratória) e métodos mais ativos como técnicas selecionadas de FNP, são bem indicados. ${ }^{10}$

Já os pacientes com dor lombar crônica beneficiam-se das técnicas de FNP, assim como voluntários saudáveis, por seus efeitos sobre o sistema muscular envolvidos na estabilização da coluna vertebral e ainda sobre o sistema cardiovascular e respiratório. ${ }^{2,29} \mathrm{~A}$ contração ativa induzida pela técnica melhora a força da musculatura abdominal. Dentre seus afeitos, não só reduz a dor lombar crônica, mas também evita sua recorrência, pois a estabilidade do tronco é melhorada. ${ }^{10,11}$

Pacientes pós-AVC isquêmico apresentaram redução da atividade da musculatura de respiração acessória após intervenção com FNP. ${ }^{13} \mathrm{E}$ em pós-cirúrgicos de ponte de safena houve melhora significativa na capacidade pulmonar total e na correlação VEF1 (volume expiratório forçado no primeiro segundo) e CVF (capacidade vital forçada). ${ }^{15}$ Essa correlação também foi encontrada em idosos saudáveis que receberam apenas a técnica de FNP. ${ }^{19}$

A reabilitação é uma parte importante do tratamento do paciente pós-AVC e cirurgias cardiovasculares e torácicas, pois auxilia na melhora da capacidade física e respiratória. Os exercícios respiratórios levam a uma redução ou eliminação completa de 
complicações pulmonares potenciais resultantes de mecânica respiratória prejudicada, aumentando a dor, tosse, retenção de catarro ou comprometimento do diafragma. ${ }^{30}$

Nos pacientes pós-AVC o tratamento com FNP também resultou em uma diminuição apreciável na atividade bioelétrica de todos os músculos acessórios da respiração investigados. ${ }^{31} \mathrm{~A}$ diminuição dizia respeito a ambos os lados afetados e não afetados pela doença. Em contrapartida, o posicionamento corporal, utilizado no estudo, por si só apresentou, na maioria dos casos, uma menor diminuição da bioatividade muscular ou apenas uma tendência de diminuição após a manobra de inspiração/expiração máxima e a potencialização da diminuição da atividade no lado afetado observada no grupo que recebeu a técnica de FNP. ${ }^{13} \mathrm{~A}$ estimulação de FNP dos músculos respiratórios deve ativar todo o tórax, tronco e cintura escapular, proporcionando relaxamento muscular e reduzindo a espasticidade muscular. ${ }^{2,13}$ Uma redução na atividade do músculo peitoral maior e em outras atividades musculares acessórias pode, portanto, fundamentar os benefícios relacionados ao FNP na função respiratória. ${ }^{13}$

Em um estudo randomizado com atletas femininas de natação, a intervenção com FNP em membros superiores não apresentou diferença significativa na PA e na FC das voluntárias, comparado aos grupos que realizaram apenas alongamento estático ou sem intervenção. ${ }^{17}$

Poucos estudos examinaram os efeitos do alongamento sobre as variáveis FC e PA. No entanto, trabalhos anteriores sugeriram que a ativação de fibras e metaborreceptores do tipo III pode inibir o ramo parassimpá- tico do sistema nervoso autônomo e a estimulação quimiorreflexa, contribuindo para o aumento da FC humana. ${ }^{32,33}$ Portanto, talvez a curta duração do período de alongamento (2x por 30 segundos) neste estudo foi insuficiente para desencadear um aumento agudo na participação do ramo simpático do sistema nervoso autônomo dos participantes. ${ }^{17}$ Entretanto, em contraste com o grupo controle, houve redução na $\mathrm{SO}_{2}$ nos grupos de FNP e alongamento estático. ${ }^{17}$ Esses resultados podem indicar uma indução de efeitos deletérios agudos para o desenvolvimento dos membros superiores nessas atletas por diminuir o aporte de oxigênio aos músculos.

Outro estudo mostrou que indivíduos saudáveis e sedentários ao realizar técnicas de FNP em polia não afetaram seus níveis de PA e FC ao final da intervenção comparado ao estado basal. ${ }^{16}$ Estudos tradicionais sobre exercícios de resistência descrevem que a maior quantidade de músculos envolvidos na atividade de resistência aumenta a resposta da pressão arterial. ${ }^{34}$ Essa resposta se deve ao maior número de artérias comprimidas pelo exercício e ao maior número de receptores periféricos estimulados. Mesmo assim, embora esses indivíduos tenham realizado o exercício com envolvimento de grande quantidade de músculos, não foi possível causar efeitos pressóricos significativos, necessitando de estudos futuros comparando exercícios com técnicas de FNP e exercícios tradicionais. ${ }^{16}$

No entanto, neste estudo, entre as séries do exercício houve alteração em PA e FC dos voluntários. ${ }^{35} \mathrm{~A}$ principal explicação para esse fenômeno é um mecanismo reflexo do sistema nervoso causado pela 
atividade muscular, receptores que aumentam sua atividade devido à estimulação mecânica, denominados mecanorreceptores ou receptor tipo III e por meio da estimulação metabólica denominada metaborreflexo ou receptor tipo IV. ${ }^{36}$ Neste estudo, a possível atividade desses receptores produziu efeitos significativos na FC e sobrecarga cardíaca por um aumento no PA. ${ }^{32}$ Além disso, é possível que esses receptores possam influenciar no aumento da percepção de esforço exercido sobre o membro pela estimulação do receptor tipo IV que também possui conexões nociceptivas. ${ }^{32,36}$

\section{CONSIDERAÇõES FINAIS}

De acordo com os estudos descritos nesta revisão de literatura, o FNP parece ser uma técnica promissora a ser utilizada na área cardiorrespiratória. Seus efeitos em estudos clínicos parecem beneficiar pacientes com sintomas cardiovasculares e respiratórios e com consequências de doenças como: doença pulmonar obstrutiva crônica, câncer, lombalgia, pós-AVC e distrofia miotônica. Além disso, seu uso na reabilitação de pacientes pós-cirúrgicos e nas disfunções cardiorrespiratórias do envelhecimento também se demonstra importante. No entanto, sua diversidade metodológica aponta para a necessidade de mais estudos, randomizados, controlados, com maior casuística e com melhor padronização para descrição e comparação de diferentes protocolos de tratamento sobre parâmetros cardiopulmonares.

\section{REFERÊNCIAS}

1. Etnyre BR, Abraham LD. H-reflex changes during static stretching and two variations of proprioceptive neuromuscular facilitation techniques. Electroencephalogr Clin Neurophysiol. 1986; 63(2):174-179

2. Victoria GD, Carmen EV, Alexandru S, Antoanela O, Florin C, Daniel D. The pnf (proprioceptive neuromuscular facilitation) stretching Technique - a brief review. Sci Mov Heal. 2013; $8(2): 623-8$

3. Silva IA, Amorim JR, Carvalho FT, Mesquita LSA. Efeito de um protocolo de Facilitação Neuromuscular Proprioceptiva (FNP) no equilíbrio postural de idosas. Fisioter. Pesqui. 2017; 24 (1): 62-7

4. Kim EK, Lee DK, Kim YM. Effects of aquatic PNF lower extremity patterns on balance and ADL of stroke patients. J Phys Ther Sci. 2015;27(1):213-5

5. Nitz J, Burke B. A study of the facilitation of respiration in myotonic dystrophy. Physiother Res Int. 2002;7(4):228-38

6. Cortopassi F, Castro AA, Porto EF, Colucci M, Fonseca G, Torre-Bouscoulet L, et al. Comprehensive exercise training improves ventilatory muscle function and reduces dyspnea perception in patients with COPD. Monaldi Arch Chest Dis. 2009;71(3):106-12.

7. de Souza GF, Castro AA, Velloso M, Silva CR, Jardim JR.Lactic acid levels in patients with chronic obstructive pulmonary disease accomplishing unsupported arm exercises. Chron Respir Dis. 2010; 7(2):75-82 
8. Velloso M, do Nascimento NH, Gazzotti MR, Jardim JR. Evaluation of effects of shoulder girdle training on strength and performance of activities of daily living in patients with chronic obstructive pulmonary disease. Int J Chron Obstruct Pulmon Dis. 2013; 8:187-192

9. Seo K, Cho M. The effects on the pulmonary function of normal adults proprioceptive neuromuscular facilitation respiration pattern exercise. J Phys Ther Sci. 2014;26(10):1579-82

10. Pyszora A, Budzyński J, Wójcik A, Prokop A, Krajnik M. Physiotherapy programme reduces fatigue in patients with advanced cancer receiving palliative care: randomized controlled trial. Support Care Cancer. 2017; 25(9):2899-908

11. Kim BR, Lee HJ. Effects of proprioceptive neuromuscular facilitation-based abdominal muscle strengthening training on pulmonary function, pain, and functional disability index in chronic low back pain patients. J Exerc Rehabil. 2017;13(4):486-90

12. Morano MT, Araújo AS, Nascimento FB, da Silva GF, Mesquita R, Pinto JS, et al. Preoperative pulmonary rehabilitation versus chest physical therapy in patients undergoing lung cancer resection: a pilot randomized controlled trial. Arch Phys Med Rehabil. 2013;94(1):53-8

13. Slupska L, Halski T, Żytkiewicz M, Ptaszkowski K, Dymarek R, Taradaj J, et al. Proprioceptive Neuromuscular Facilitation for Accessory Respiratory Muscles Training in Patients After Ischemic Stroke. Adv Exp Med Biol. 2019; 1160:81-91

14. Shiwa SR, Costa LOP, Moser ADL, Aguiar IC, Oliveira LVF. PEDro: a base de dados de evidências em fisioterapia. Fisioter em Mov. 2011; 24(3):523-33

15. Bujar-Misztal M, Chciałowski A. Influence of Proprioceptive Neuromuscular Facilitation on Lung Function in Patients After Coronary Artery Bypass Graft Surgery. Adv Exp Med Biol. 2018;1116:11-17

16. Silva AA, Lobato AN, Pereira EN, Silva FS, Freire RC Júnior, Arêas FZS, et al. Cardiovascular and relative exertion perceived response during proprioceptive neuromuscular facilitation exercise associated to pulley. Man. Ther., Posturology Rehabil. J. 2014; 12: 358-63

17. Silva GC, Di Masi F, Paixão A, Bentes CM, Sá M, Miranda H, et al. Effects of Proprioceptive Neuromuscular Facilitation Stretching and Static Stretching on Cardiovascular Responses. J Exerc Physiol Online. 2013; 16: 117-25

18. Reddy SV, Arumugam G, Ajin, Kumar R, Jose N. Determine the significance of proprioceptive neuromuscular facilitation technique with conventional chest physiotherapy in mechanically ventilated organophosphorus poisoning patients. Indian J Med Res. 2014; 3(2): 461-6

19. BR G, Goud A. Short term effects of respiratory muscle stretch gymnastics versus hold relax PNF on pulmonary functions and chest expansion in elderly individuals-a randomized clinical trial. Int J Appl Res 2017;3(7):1018-22

20. Moreno MA, Silva E, Zuttin RS, Gonçalves M. Effect of a training program based on proprioceptive neuromuscular facilitation onto thoracic mobility. Fisioter e Pesqui [Internet]. 2009 [Acesso em: 2021 abr 09]; 16: 161-5. Disponível em: http://www.scielo.br/scielo.php?script=sci_ arttext\&pid=S1809-29502009000200012\&lang=pt\%0Ahttp://www.scielo.br/pdf/fp/v16n2/12. pdf.

21. Machuca-Tzili L, Brook D, Hilton-Jones D. Clinical and molecular aspects of the myotonic dystrophies: a review. Muscle Nerve. 2005;32(1):1-18

22. Allaire J, Maltais F, Doyon JF, Noël M, LeBlanc P, Carrier G, et al. eripheral muscle endurance and the oxidative profile of the quadriceps in patients with COPD. Thorax. 2004;59(8):673-8

23. Gea JG, Pasto M, Carmona MA, Orozco-Levi M, Palomeque J, Broquetas J. Metabolic characteristics of the deltoid muscle in patients with chronic obstructive pulmonary disease. Eur Respir J. 2001;17(5):939-45 
24. Castagna O, Boussuges A, Vallier JM, Prefaut C, Brisswalter J. Is impairment similar between arm and leg cranking exercise in COPD patients? Respir Med. 2007; 101(3):547-53.

25. Sánchez-Santed F, Colomina MT, Herrero Hernández E. Organophosphate pesticide exposure and neurodegeneration. Cortex. 2016; 74:417-26

26. Sidhu GK, Singh S, Kumar V, Dhanjal DS, Datta S, Singh J. Toxicity, monitoring and biodegradation of organophosphate pesticides: A review. Crit Rev Environ Sci Technol. [Internet] 2019 [Acesso em: 2021 abr 09]; 49(13): 1135-87. Disponível em: https://doi.org/10.1080/10643389.2 019.1565554

27. Hulzebos EH, Helders PJ, Favié NJ, De Bie RA, Brutel de la Riviere A, Van Meeteren NL. Preoperative intensive inspiratory muscle training to prevent postoperative pulmonary complications in high-risk patients undergoing CABG surgery: a randomized clinical trial. JAMA. 2006;296(15):1851-7

28. Dong ST, Butow PN, Costa DS, Lovell MR, Agar M. Symptom clusters in patients with advanced cancer: a systematic review of observational studies. J Pain Symptom Manage [Internet]. 2014 [Acesso em: 2021 abr 09]; 48(3):411-450. Disponível em: http://dx.doi.org/10.1016/j.jpainsymman.2013.10.027

29. Balcı NC, Yuruk ZO, Zeybek A, Gulsen M, Tekindal MA. Acute effect of scapular proprioceptive neuromuscular facilitation (PNF) techniques and classic exercises in adhesive capsulitis: a randomized controlled trial. J Phys Ther Sci. 2016; 28(4):1219-27

30. Efthimiou J, Butler J, Woodham C, Benson MK, Westaby S. Diaphragm paralysis following cardiac surgery: role of phrenic nerve cold injury. Ann Thorac Surg. [Internet]. 1991 [Acesso em: 2021 abr 09]; 52(4):1005-8. Disponível em: http://dx.doi.org/10.1016/0003-4975(91)91268-Z

31. Khedr EM, El Shinawy O, Khedr T, Aziz Ali YA, Awad EM. Assessment of corticodiaphragmatic pathway and pulmonary function in acute ischemic stroke patients. Eur J Neurol. 2000; 7(3):323-30

32. Coote JH, Bothams VF. Cardiac vagal control before, during and after exercise. Exp Physiol. 2001; 86(6):811-5

33. Kaufman MP, Hayes SG. The exercise pressor reflex. Clin Auton Res. 2002; 12(6):429-39

34. Barclay CJ. Energetics of contraction. Compr Physiol. 2015; 5(2):961-95

35. Cunha LM, Duarte RCB, Silva LCS. Investigação clínica, bioquímica e genética de pacientes do Norte do Brasil com adrenoleucodistrofia ligada ao cromossomo X. Neurociencias. 2008; 4: 107-10.

36. Ray CA, Carter JR. Central modulation of exercise-induced muscle pain in humans. J Physiol. 2007; 585(Pt 1):287-294

\section{DAdos DOS AUTORES}

\section{Gustavo Silveira da Silva}

Doutorando do departamento de imunologia do Instituto de Ciências Biomédicas da Universidade de São Paulo (USP), São Paulo/SP - Brasil. gustavoss_1@hotmail.com

\section{Natanyelle Steffen Freiberger}

Fisioterapeuta formada pela Universidade do Sul de Santa Catarina (Unisul), Palhoça/SC Brasil.nelly_freiberger1@hotmail.com

\section{Nathália Cristina Oliveira Teodoro}

Fisioterapeuta formada pela Universidade do Sul de Santa Catarina (Unisul), Palhoça/SC Brasil.nathalia_cris-oliveira@hotmail.com 


\section{Fernanda Machado}

Fisioterapeuta formada pela Universidade do Sul de Santa Catarina (Unisul), Palhoça/SC - Brasil. fernandamachado525@gmail.com

\section{Karoliny dos Santos Isoppo}

Docente do curso de Fisioterapia da Universidade do Sul de Santa Catarina (Unisul), Palhoça/SC - Brasil. fisio.karoliny@gmail.com

Submetido em: 19-3-2021

Aceito em: 17-5-2021 